GABRIELA VIRGINIA MOREIRA

\title{
LIRAGLUTIDA PROMOVE MUDANÇA DA MICROBIOTA INTESTINAL COM REDUÇÃO DA MASSA ADIPOSA E DA ESTEATOSE HEPÁTICA NÃO- ALCÓOLICA EM DOIS MODELOS ANIMAIS DE OBESIDADE.
}

Tese apresentada ao Programa de PósGraduação em Fisiologia Humana e Biofisica no Instituto de Ciências Biomédicas da Universidade de São Paulo, para obtenção do título de Doutora em Ciências.

Área de Concentração: Fisiologia Humana

Orientadora: $\operatorname{Prof}^{\circ} \operatorname{Dr}^{\circ}$ Carla Roberta de Oliveira Carvalho

Versão corrigida. A versão original eletrônica, encontra-se disponível tanto na Biblioteca do ICB quanto na Biblioteca Digital de Teses e Dissertações da USP (BDTD). 


\section{RESUMO}

Moreira GV. Liraglutida promove mudança da microbiota intestinal com redução da massa adiposa e da esteatose hepática não-alcóolica em dois modelos animais de obesidade. [Tese (Doutorado em Fisiologia Humana)]. São Paulo; Instituto de Ciências Biomédicas, Universidade de São Paulo; 2017.

O presente estudo analisou os efeitos do análogo do GLP-1, liraglutida, na composição da flora intestinal e perda de peso de dois modelos de obesidade: um induzido por dieta hiperlipidica (HFD) e outro de obesidade genética (camundongos $o b / o b)$. Para tanto, os dois modelos de obesidade foram tratados com liraglutida durante duas semanas. Os perfis metabólicos foram feitos por meio de testes glicêmicos e insulínicos, análise histológica do fígado, região cecal e dos coxins gordurosos, ingestão alimentar, peso corporal e análise metagenômica da região $16 \mathrm{~s}$ rRNA de bactérias do conteúdo cecal. O tratamento induziu perda de peso significativa nos dois modelos estudados, com melhora dos níveis glicêmicos e redução de células inflamatórias na região cecal e do fígado, assim como promoveu o aumento de células caliciformes epiteliais. Nos camundongos ob/ob, o tratamento com a liraglutida foi capaz de reduzir em $78 \%$ o acúmulo de gordura hepática promovendo a redução da doença gordurosa hepática não alcoólica (DGHNA) associada a redução do infiltrado inflamatório nos espaços porta hepáticos, portanto reduzindo a esteato-hepatite não alcoólica (EHNA). As análises da microbiota intestinal mostraram que liraglutida mudou a estrutura geral taxonômica entre os grupos experimentais, bem como a abundância relativa de elementos bacterianos já sabidamente envolvidos com peso e controle glicêmico como redução de Proteobacterias e aumento de Akkermansia muciniphila no grupo HFD. Apresentamos a primeira evidência da ação da liraglutida induzindo atenuação da DGHNA de EHNA a simples esteatose hepática e perda de peso associados às mudanças da microbiota intestinal. Além do mais, esse estudo gerou uma nova lista de potenciais alvos bacterianos que podem interferir positivamente com o metabolismo energético na direção do controle clinico dessas doenças metabólicas.

Palavras-chave: Liraglutida. Análogo GLP-1. Microbiota intestinal. Obesidade. EHNA. 


\begin{abstract}
Moreira GV. Liraglutide changes gut microbiota and reduces hepatic steatosis and fat mass in two models of obesity mice. [Ph. D. thesis (Human Physiology)]. São Paulo: Instituto de Ciências Biomédicas, Universidade de São Paulo; 2017.

The present study was performed to analyze the effects of liraglutide on the composition of the gut microbiota and weight-loss in two obesity model: obesity induced by high fat diet (HFD) and genetic obese mice $(o b / o b)$. Both models of obesity mice were treated with liraglutide for two weeks. Metabolic profiles were measured by glycemic and insulin test, histological liver, cecal region and fat pad morphologies were analyzed, food intake, body weight and metagenomic analysis of the region 16s rRNA of bacteria cecal contents. The treatment induced significant weight-loss in both obesity model with improvement of glycemic parameters and reduction of inflammatory cells in the cecum and the liver, as well as promoted the increase of epithelial goblet cells in animals with induced obesity. In ob/ob mice the liraglutide treatment was able to reduce by $78 \%$ the accumulation of liver fat thereby reducing the inflammatory nonalcoholic hepatic steatosis frame (NASH). The gut microbiota analyzes showed that liraglutide changed the overall structure as well the relative abundance of weightrelevant phylotypes as reduction of Proteobacteria and increases of Akkermansia muciniphila in treated HFD group. We present the first evidence of the action of liraglutide leading to the improvement of $\mathrm{NASH}$ and weight loss associated with changes in the intestinal microbiota. Moreover, by the profile of the intestinal microbiota, we present a list of potential bacterial targets that may affect the metabolism inducing a more similar metabolic profile to that considered normal or clinically controlled.
\end{abstract}

Keywords: Liraglutide. GLP-1 analogue. Gut microbiome. Obesity and NASH. 


\section{INTRODUÇÃO}

O plano de ações estratégicas para o combate de doenças crônicas do Ministério da Saúde destaca diabetes, cardiopatias e acidente vascular encefálico como as principais doenças que levarão a perda econômica brasileira de US $\$ 4.18$ bilhões entre os anos de 2006 a $2015{ }^{1}$.

O último relatório da Organização Mundial de Saúde (OMS) revela que mais de 16 milhões de brasileiros adultos $(8,1 \%)$ portadores de diabetes, sendo a responsável pela morte de 72 mil pessoas por ano. O relatório também revela que 422 milhões de adultos em todo o mundo viviam com diabetes em 2014, quatro vezes mais do que em $1980^{2}$.

Uma das principais causas atreladas à fatores de riscos metabólicos são o excesso de peso e a obesidade, que segundo o mesmo órgão, no Brasil, acomete $54 \%$ e $20 \%$ da população respectivamente ${ }^{3}$.

Atualmente, um dos tratamentos disponíveis para o tratamento de pacientes com diabetes tipo 2 está sendo estudado como uma alternativa para o tratamento da obesidade devido seus efeitos colaterais.

A liraglutida é uma fármaco análogo ao GLP-1(glucagon like peptide-1), com 97\% de homologia com o GLP-1 humano, incluindo uma mutação de lisina para arginina na posição 34 e uma cadeia lateral palmitoil na lisina 264 .

O GLP-1 é uma incretina secretada pelas células $L$ da mucosa intestinal. Atua sobre as células das ilhotas pancreáticas induzindo a liberação de insulina pelas células $\beta$ e a redução de glucagon pelas células $\alpha$, em resposta à ingestão de alimentos ${ }^{5}$. A ativação do receptor de GLP-1 (GLP-1R) induz a expressão de proteínas e genes que aumentam a proliferação e diminuem a apoptose das células $\beta$ por diferentes vias de sinalização que culminam com a ativação das proteínas ERK1/2 6,7.

Estudos demostraram que o GLP-1 é capaz de reprogramar as células $\beta$ pancreáticas defeituosas tornando-as mais sensíveis à glicose ${ }^{8}$; sendo que sua administração subcutânea em paciente com DM do tipo 2 promove a redução da glicemia e da HbA1c tanto no jejum quanto pós-prandial, além de promover a redução do peso corpóreo a longo prazo. ${ }^{8,9}$.

A resistência à insulina ${ }^{10} \mathrm{e}$ a obesidade ${ }^{11}$ são alguns dos fatores que resultam em defeitos na liberação do GLP-1. Essas alterações metabólicas promovem o menor estimulo do pâncreas para a biossíntese e sensibilidade à insulina, assim como o 
aumento da secreção de glucagon, do esvaziamento gástrico e por consequência da ingestão alimentar ${ }^{10-14}$. Diante disso, o GLP-1 facilita a rápida depuração e armazenamento de glicose no sangue ${ }^{15}$.

Outros efeitos do GLP-1, como regulação da alimentação, motilidade gástrica, glicorregulação e função cardiovascular, são mediados pelo $X$ par de nervos cranianos, o nervo vago e seu homólogo contralateral, que modulam atividades nos tecidos hepático, adiposo, muscular e pâncreas ${ }^{11,16}$.

Embora o GLP-1 endógeno tenha uma diversidade de efeitos fisiológicos, é rapidamente inativado, tendo uma meia-vida de menos de 2 minutos, por ação da enzima dipeptidil-peptidade-4 (DPP-IV) ${ }^{17}$. A liraglutida, atualmente utilizada para tratamento do DM do tipo 2, possui resistência a degradação enzimática da DPP-IV, o que aumenta a meia-vida desse mimético hormonal na circulação por até 8 horas ${ }^{18}$

Recentes estudos sugerem um papel importante do GLP-1 e de sua atuação em células do epitélio intestinal, correlacionando seus efeitos a mudanças na flora intestinal ${ }^{19,20}$. Relacionam a fermentação produzida por bactérias intestinais com a produção de ácidos graxos de cadeia curta (AGCC) e a secreção/regulação de hormônios peptídicos ${ }^{21}$ e principalmente de GLP-1 pelas células $L$ intestinais ${ }^{22-24}$.

Apesar das diversas evidências sobre os análogos do GLP-1, até agora não estão claros quais os fatores que contribuem para seus efeitos adipogênicos e se seus efeitos protetores possuem alguma relação com alterações da microbiota.

\subsection{Microbiota intestinal}

A flora intestinal é composta por aproximadamente 1.000 a 1.150 espécies diferentes de bactérias ${ }^{25}$ capazes de viver de modo comensal com seu hospedeiro, comunicando-se entre si por meio de transformações químicas fisiologicamente importantes ${ }^{26}$.

O papel da microbiota intestinal vem sendo reconhecido como um dos maiores fatores ambientais responsáveis por modificações metabólicas no organismo de seu hospedeiro ${ }^{27}$.

Em 2006, em um dos primeiros estudos relacionando microbiota intestinal com regulação metabólica, Turnbaugh et al. demonstraram que a transfecção da flora intestinal de camundongos obesos para camundongos germ-free, resultava em aumento da massa corpórea total quando comparados aos colonizados com 
microbiota de animais magros. Os dados foram obtidos por meio da caracterização da microbiota intestinal de camundongos obesos (ob/ob) e seus controles magros (ob/+ e +/+). Naquele estudo constataram que os animais obesos possuem maior proporção de bactérias intestinais do filo Firmicutes enquanto que os magros possuem maior proporção de bactérias do filo Bacteroidetes ${ }^{28}$. Resultados semelhantes foram observados com a manipulação da microbiota intestinal via antibiótico com ação inibitória sobre a síntese da parede celular bacteriana, a vancomicina, em pacientes obesos por 7 dias. Foi demonstrada redução da diversidade bacteriana e, em particular redução da abundância de Firmicutes, filo bacteriano envolvido com a produção de AGCC e ácidos biliares ${ }^{29}$.

Estudos do epitélio intestinal de camundongos germ-free mostram que os mesmos são mais suscetíveis às infecções, possuem a vascularização e a atividade de enzimas digestivas reduzidas e aumento nos níveis séricos de citocinas. No entanto, a reconstituição com uma microflora intestinal é suficiente para restaurar o sistema imunológico ${ }^{30}$.

Durante a digestão da gordura no trato gastrointestinal pode haver desencadeamento de respostas inflamatórias devido a ligação de componentes lipídicos da microbiota, como os lipopolissacarídeos, LPS, aos receptores do tipo Toll Like Receptors (TLR) presentes em células do sistema imunológico. Esses receptores estão envolvidos no reconhecimento de patógenos ${ }^{31}$. A função fisiológica desses TLR é sinalizarem para manutenção da barreira intestinal contra possível infiltração de patógenos, ou seja impedir infecção ${ }^{32}$.

Novos estudos levantam a hipótese de que lipídios específicos das dietas atuais são reconhecidos pelo nosso sistema imunológico inato, mais especificamente pelo TLR4, como intrusos, semelhante a agentes patogênicos bacterianos. Estes TLR4 em macrófagos e tecido adiposo são induzidos a produzir citocinas próinflamatórias, que por sua vez levam a resistência à insulina principalmente nos tecido muscular esquelético ${ }^{33}$.

Cani et al., (2008) também demonstraram que o tratamento com antibióticos reduz os níveis plasmáticos de LPS, a permeabilidade intestinal, os depósitos gordurosos viscerais e a infiltração por macrófagos em animais alimentados com dieta hiperlipídica. 
Sabe-se que o tecido adiposo ${ }^{35}$ e o intestino ${ }^{36}$ possuem um papel central na secreção de inúmeros mediadores químicos que modulam o apetite, a ação da insulina e mesmo respostas inflamatórias.

Caricilli et al., (2011) analisaram a microbiota intestinal como um modulador da resistência à insulina em camundongos nocaute para o TLR2. Observou-se que esses animais possuíam um fenótipo semelhante ao da síndrome metabólica e com uma composição bacteriana similar à encontrada em indivíduos obesos, ou seja, com aumento da proporção de bactérias do filo Firmicutes. Essa modulação da flora intestinal estaria associada com aumentos de LPS plasmático, resistência à insulina e diabetes, acumulo de triglicérides no fígado e da gordura corpórea.

A atividade metabólica bacteriana extrai substratos dos alimentos ingeridos que serão absorvidos fornecendo energia e nutrientes para o crescimento e proliferação bacteriana e do hospedeiro em questão ${ }^{38,39}$. Essa atividade metabólica se mostra importante quando comparamos camundongos germ-free, que requerem maior ingestão de calorias para manter um peso corporal normal ${ }^{26,30}$. Diante disto podemos pressupor que bactérias intestinais atuem como moduladores da deposição de gordura no hospedeiro.

Os mecanismos envolvidos na obesidade de animais ob/ob são diferentes dos envolvidos na obesidade por dieta. Camundongos ob/ob possuem mutação no cromossomo 6 que leva a ausência de leptina ou à presença de leptina não funcional, tornando-os ao fenótipo de resistência à insulina, hiperfagia e obesidade ${ }^{40}$. Os receptores de leptina desempenham papéis fundamentais no controle do apetite e na regulação do consumo de energia da mucosa gástrica de roedores e humanos. No tecido adiposo a secreção endócrina de leptina ocorre de forma lenta, e atua como um sinalizador ao sistema nervoso central, sobre o aporte energético do organismo e controle em longo prazo da ingestão de alimentos, gasto de energia e metabolismo da glicose nos tecidos periféricos. Por outro lado, a leptina também pode ser produzida e secretada por outros órgãos como a mucosa gástrica do estomago. Nesse órgão a secreção exócrina ocorre de forma rápida. A leptina chega ao duodeno ativando os receptores que se encontram na mucosa intestinal. Estes receptores estão envolvidos no controle da absorção de nutrientes pelos enterócitos, na secreção de muco pelas células caliciformes, na motilidade intestinal e na produção de peptídeos do tipo glucagon 1 e 2 (GLP-1 e GLP-2) que inibem o esvaziamento gástrico ${ }^{36}$. 
Frente as recentes publicações na área de doenças de metabólicas, focar na compreensão do papel do trato gastrointestinal na regulação do equilíbrio energético do corpo e da função metabólica mostra-se cada vez mais importante para entender a miríade de respostas à resistência insulínica e obesidade.

Embora esteja bem estabelecido que a liraglutida melhora os parâmetros da homeostase de glicose, o papel potencial da liraglutida na obesidade, nos distúrbios hepáticos associados, na inflamação metabólica e na microbiota intestinal são desconhecidos.

Levando em consideração o exposto, nossa hipótese é que o efeito colateral da liraglutida sobre a obesidade de pacientes diabéticos tipo 2 poderia ser decorrente da modulação da flora intestinal. Para tanto, foram utilizados dois modelos animais de obesidade, um induzido por uma dieta hiperlipidica e o outro devido a alterações genéticas, ob/ob, com a finalidade de identificar possíveis Filos bacterianos comuns sob efeito da liraglutida.

\subsection{Hipótese}

O uso de análogos do GLP-1 atua sobre obesidade induzida via dietas obesogênicas e obesidade genética modulando a flora intestinal. 


\section{CONCLUSÃO}

O presente estudo confirmou a interação molecular e metabólica microbiotahospedeiro. Além disso, demonstrou que o análogo do GLP-1, liraglutida, atua modulando as populações bacterianas em camundongos obesos e magros. Ademais, essa mudança está associada a melhora dos parâmetros glicêmicos, inflamatórios, atenuação da DGHNA - modificando quadro de esteato-hepatite (EHNA) a simples esteatose - e perda de peso em dois diferentes modelos de obesidade: um induzido por dieta rica de lipídios - e hiperleptinêmico - e outro modelo de obesidade genética - hipoleptinêmico.

O resultado da análise metagenômica da microbiota intestinal nos forneceu uma nova lista de potenciais alvos bacterianos que podem afetar o metabolismo em situações de obesidade e de EHNA. Abrindo campo extenso de investigação científica para avaliar os mecanismos de celulares de sinalização envolvidos.

E, por fim, esse estudo possui relevância clinica uma vez que demonstra efeito do uso da liraglutida a mais do que sua indicação. Esse fármaco pode minimizar ou reverter a esteato-hepatite não alcoólica (EHNA), provavelmente pela modulação da diversidade da microbiota intestinal. 


\section{REFERÊNCIAS *}

1. Malta DC, Morais Neto OL De, Silva Junior JB Da. Apresentação do plano de ações estratégicas para o enfrentamento das doenças crônicas não transmissíveis no Brasil, 2011 a 2022. Epidemiol e Serviços Saúde 2011; 20: 425-438.

2. WHO. Infobase global comparable estimates, risk factors: prevalence of diabeteshttp://www.portal.pmnch.org/diabetes/global-report/en/ (2016).

3. WHO. Infobase global comparable estimates, risk factors: prevalence of overweight and obesity maphttp://www.portal.pmnch.org/diabetes/global-report/en/ (2015).

4. Knudsen LB, Nielsen PF, Huusfeldt PO, et al. Potent derivatives of glucagon-like peptide-1 with pharmacokinetic properties suitable for once daily administration. J Med Chem 2000; 43: 16641669.

5. Rask E, Olsson T, Söderberg S, et al. Impaired incretin response after a mixed meal is associated with insulin resistance in nondiabetic men. Diabetes Care 2001; 24: 1640-1645.

6. Forti ACE. Estratégias terapêuticas baseadas nas vias do GLP-1. Johns Hopkins Adv Stud Med 2006; 6: 618-626.

7. Quoyer J, Longuet C, Broca C, et al. GLP-1 Mediates Antiapoptotic Effect by Phosphorylating Bad through a -Arrestin 1-mediated ERK1/2 Activation in Pancreatic -Cells. J Biol Chem 2010; 285: 1989-2002.

8. Zander M, Madsbad S, Madsen JL, et al. Effect of 6-week course of glucagon-like peptide 1 on glycaemic control, insulin sensitivity, and $\beta$-cell function in type 2 diabetes: a parallel-group study. Lancet 2002; 359: 824-830.

9. Fujishima Y, Maeda N, Inoue K, et al. Efficacy of liraglutide, a glucagon-like peptide-1 (GLP-1) analogue, on body weight, eating behavior, and glycemic control, in Japanese obese type 2 diabetes. Cardiovasc Diabetol 2012; 11: 107.

10. Holst JJ. On the physiology of GIP and GLP-1. Horm Metab Res 2004; 36: 747-754.

11. Drucker DJ. Glucagon-Like Peptide-1 and the Islet-Cell: Augmentation of Cell Proliferation and Inhibition of Apoptosis. Endocrinology 2003; 144: 5145-5148.

12. Huang $Y$, Wilkinson $G$, Willars $G$. Role of the signal peptide in the synthesis and processing of the glucagon-like peptide-1 receptor. Br J Pharmacol 2010; 159: 237-251.

13. Donato J, Frazão R, Elias CF. The PI3K signaling pathway mediates the biological effects of leptin. Arq Bras Endocrinol Metab 2010; 54: 591-602.

14. $\mathrm{Li} \mathrm{Y}$, Tweedie D, Mattson MP, et al. Enhancing the GLP-1 receptor signaling pathway leads to proliferation and neuroprotection in human neuroblastoma cells. J Neurochem 2010; 113: 16211631.

15. Hayes MR, Kanoski SE, Alhadeff AL, et al. Comparative effects of the long-acting GLP-1 receptor ligands, liraglutide and exendin-4, on food intake and body weight suppression in rats. Epub

\footnotetext{
*De acordo com:

International Committee of Medical Journal Editors. [Internet]. Uniform requirements for manuscripts submitted to Biomedical Journal: sample references. updated 2011 Jul 15]. Available from: http://www.icmje.org
} 
ahead of print 2013. DOI: doi:10.1038/oby.2011.50.

16. Baggio LL, Drucker DJ. Biology of Incretins: GLP-1 and GIP. Gastroenterology 2007; 132: 21312157.

17. Neumiller JJ. Differential chemistry (structure), mechanism of action, and pharmacology of GLP1 receptor agonists and DPP-4 inhibitors. In: J Am Pharm Assoc (2003). Department of Pharmacotherapy, College of Pharmacy, Washington State University, Spokane, WA 99217, USA. jneumiller@wsu.edu, pp. S16-29.

18. Drucker DJ, Dritselis A, Kirkpatrick P. Liraglutide. Nat Rev Drug Discov 2010; 9: 267-268.

19. Cani PD, Everard A, Duparc T. Gut microbiota, enteroendocrine functions and metabolism. Curr Opin Pharmacol 2013; 13: 935-940.

20. Hwang I, Park YJ, Kim Y-R, et al. Alteration of gut microbiota by vancomycin and bacitracin improves insulin resistance via glucagon-like peptide 1 in diet-induced obesity. FASEB J 2015; $1-15$.

21. Everard A, Cani PD. Gut microbiota and GLP-1. Reviews in Endocrine and Metabolic Disorders, 2014, pp. 189-196.

22. Tsuda T. Possible abilities of dietary factors to prevent and treat diabetes via the stimulation of glucagon-like peptide-1 secretion. Mol Nutr Food Res 2015; n/a-n/a.

23. Dalgaard M, Thomsen $\mathrm{C}$, Rasmussen BM, et al. Ethanol with a mixed meal decreases the incretin levels early postprandially and increases postprandial lipemia in type 2 diabetic patients. Metabolism 2004; 53: 77-83.

24. Iwasaki M, Hoshian F, Tsuji T, et al. Predicting efficacy of dipeptidyl peptidase-4 inhibitors in patients with type 2 diabetes: Association of glycated hemoglobin reduction with serum eicosapentaenoic acid and docosahexaenoic acid levels. J Diabetes Investig 2012; 3: 464-467.

25. Qin J, Li R, Raes J, et al. A human gut microbial gene catalog established by metagenomic sequencing. 2013; 464: 59-65.

26. Bäckhed F, Ley RE, Sonnenburg JL, et al. Host-bacterial mutualism in the human intestine. Science 2005; 307: 1915-1920.

27. Tilg H, Cani PD, Mayer EA. Gut microbiome and liver diseases. Gut 2016; 65: 2035-2044.

28. Turnbaugh PJ, Ley RE, Mahowald MA, et al. An obesity-associated gut microbiome with increased capacity for energy harvest. Nature 2006; 444: 1027-1031.

29. Reijnders D, Goossens GH, Hermes GDA, et al. Effects of Gut Microbiota Manipulation by Antibiotics on Host Metabolism in Obese Humans: A Randomized Double-Blind PlaceboControlled Trial. Cell Metab 2016; 24: 63-74.

30. Shanahan F. The host-microbe interface within the gut. Bailliere's Best Pract Res Clin Gastroenterol 2002; 16: 915-931.

31. Velloso L a., Folli F, Saad MJ. TLR4 at the crossroads of nutrients, gut microbiota and metabolic inflammation. Endocr Rev 2015; er.2014-1100.

32. Wells JM, Rossi O, Meijerink M, et al. Epithelial crosstalk at the microbiota-mucosal interface. Proc Natl Acad Sci U S A 2011; 108 Suppl: 4607-4614.

33. Shi H, Kokoeva M V, Inouye K, et al. TLR4 links innate immunity and fatty acid - induced insulin 
resistance. J Clin Invest 2006; 116: 3015-3025.

34. Cani PD, Bibiloni R, Knauf C, et al. Changes in Gut Microbiota Control Metabolic EndotoxemiaInduced Inflammation in High-Fat Diet-Induced Obesity and Diabetes in Mice. Epub ahead of print 2008. DOI: 10.2337/db07-1403.

35. Kahn SE, Hull RL, Utzschneider KM. Mechanisms linking obesity to insulin resistance and type 2 diabetes. Nature 2006; 444: 840-846.

36. Cammisotto PG, Levy É, Bukowiecki LJ, et al. Cross-talk between adipose and gastric leptins for the control of food intake and energy metabolism. Prog Histochem Cytochem 2010; 45: 142200.

37. Caricilli AM, Picardi PK, de Abreu LL, et al. Gut microbiota is a key modulator of insulin resistance in TLR 2 knockout mice. In: PLoS Biol. Department of Internal Medicine, State University of Campinas, Campinas, Brazil., p. e1001212.

38. Ley RE, Bäckhed F, Turnbaugh P, et al. Obesity alters gut microbial ecology. Proc Natl Acad Sci U S A 2005; 102: 11070-11075.

39. Asselin C, Gendron FP. Shuttling of information between the mucosal and luminal environment drives intestinal homeostasis. FEBS Letters, 2014, pp. 4148-4157.

40. Marques-Lopes I, Marti A, Moreno-Aliaga MJ, et al. Aspectos genéticos da obesidade. Revista de Nutricao 2004; 17: 327-338. 